\title{
Autonomic Evaluation of Patients With Gastroparesis and Neurostimulation: Comparisons of Direct/Systemic and Indirect/Cardiac Measures
}

\author{
Abigail Stocker ${ }^{\mathrm{a}}$, Thomas L. Abell ${ }^{\mathrm{a}, \mathrm{c}}$, Hani Rashed ${ }^{\mathrm{a}}$, Archana Kedar ${ }^{\mathrm{a}}$, \\ Ben Boatright ${ }^{\mathrm{a}}$, Jiande Chen ${ }^{\mathrm{b}}$
}

\begin{abstract}
Background: Disorders of nausea, vomiting, abdominal pain, and related problems often are manifestations of gastrointestinal, neuromuscular, and/or autonomic dysfunction. Many of these patients respond to neurostimulation, either gastric electrical stimulation or electroacupuncture. Both of these therapeutic techniques appear to influence the autonomic nervous system which can be evaluated directly by traditional testing and indirectly by heart rate variability.
\end{abstract}

Methods: We studied patients undergoing gastric neuromodulation by both systemic autonomic testing ( 39 patients, six males and 33 females, mean age 38 years) and systemic autonomic testing and heart rate variability ( 35 patients, seven males and 28 females, mean age 37 years) testing before and after gastric neuromodulation. We also performed a pilot study using both systemic autonomic testing and heart rate variability in a small number of patients (five patients, all females, mean age 48.6 years) with diabetic gastroparesis at baseline to compare the two techniques at baseline. Systemic autonomic testing and heart rate variability were performed with standardized techniques and gastric electrical stimulation was performed as previously described with electrodes implanted serosally in the myenteric plexus.

Results: Both systemic autonomic testing and heart rate variability measures were often abnormal at baseline and showed changes after gastric neuromodulation therapy in two groups of symptomatic patients. Pilot data on a small group of similar patients with systemic automatic nervous measures and heart rate variability showed good concordance between the two techniques.

Conclusions: Both traditional direct autonomic measures and indirect measures such as heart rate variability were evaluated, including a pilot study of both methods in the same patient group. Both appear to be

Manuscript accepted for publication September 18, 2015

aDivision of Gastroenterology, Hepatology and Nutrition, University of Louisville, Louisville, KY, USA

bivision of Gastroenterology and Hepatology, Department of Medicine, Johns Hopkins University, Baltimore, MD, USA

${ }^{b}$ Corresponding Author: Thomas L. Abell, Division of Gastroenterology, Hepatology and Nutrition, University of Louisville, 550 S Jackson St, ACB 3L1, Louisville, KY 40202, USA. Email: thomas.abell@louisville.edu

doi: http://dx.doi.org/10.14740/gr667w useful in evaluation of patients at baseline and after stimulation therapies; however, a future full head-to-head comparison is warranted.

Keywords: Gastroparesis; Autonomic nervous system; Heart rate variability

\section{Introduction}

Background on autonomic evaluation of patients with gastroparesis undergoing neuromodulation therapies

In clinical studies, gastric electrical stimulation or gastric neuromodulation has demonstrated a potent ability to decrease nausea and vomiting in highly symptomatic idiopathic and diabetic gastoparesis patients; however, the mechanism of action remains unknown [1-5]. Proposed mechanisms of neuromodulation of the gut include central, autonomic or enteric effects. The autonomic component can be evaluated directly using autonomic nervous system (ANS) testing or indirectly using cardiac heart rate variability.

The ANS provides integration between the central nervous and enteric nervous systems by mediating reflex activities for the maintenance of homeostasis. A perturbation of the neuroendocrine and neuro-electrical microenvironment of the enteric region will manifest within the ANS. Gastroparesis is a heterogeneous disorder; however, in severe subsets, full-thickness tissue biopsies have revealed enteric neuronal and interstitial cells of Cajal histopathology [6-8], and pathologies involving extrinsic neuronal structures, as well as abnormal autonomic manifestations. Both cholinergic and adrenergic abnormalities have been reported in patients with chronic nausea and vomiting, including gastroparesis [9-13]. Heart rate variability is an indirect measure of the ANS via the cardiac pathway that can be quite useful in assessing ANS status. The low-frequency power of the heart rate variability spectrum reflects baroreflex function [14]. Heart rate variability has also been proposed as a surrogate measure for evaluating the ANS in patients with the symptoms of gastroparesis.

\section{Methodology of gastric neuromodulation}

Electrode placement for implanted gastric electrical stimulation 
utilizes two electrodes secured within the myenteric plexus: the pulse-generated electrical energy comprising gastric neuromodulation discharges into the surrounding tissue layers of the myenteric and submucosal plexus regions of extensive neuronal meshing between enteric and autonomic-afferent signaling.

A new and innovative approach to treating often debilitating symptoms is electroacupuncture as a non-implanted and truly noninvasive therapy. This was demonstrated in the transcutaneous electroacupuncture study in which 18 diabetic gastroparesis patients participated in a blinded, cross-over study in which electrostimulation (ES) and sham-ES were administered through surface electrodes at acupoints PC6 and ST36 for $2 \mathrm{~h}$ following lunch and dinner. Patients were able to apply ES/sham-ES at home using a digital microstimulator followed by in-office recordings. Patients receiving ES showed improvement in five of nine symptom scores and body pain as well as an increase in percentage of normal slow waves and vagal activity. This same approach was utilized in a pilot study of five patients.

Autonomic monitoring, routinely performed with many of our gastroparesis patients, provided a rich data source for analysis. Such an analysis was made possible using data from autonomic function testing of patients enrolled in US Food and Drug Administration-registered clinical protocols for gastric neuromodulation trials at the University of Tennessee Health Science Center (UTHSC-Memphis, TN) as center one, or at the University of Arkansas for Medical Sciences (UAMS-Little Rock, AR) as center two.

The use of transcutaneous electroacupuncture included a pilot evaluation of five patients with diabetic gastroparesis at baseline where all five patients underwent baseline systemic autonomic testing and heart rate variability testing at the University of Mississippi as center three.

\section{Patients and Methods}

\section{Autonomic function testing}

Two different approaches to autonomic testing were utilized: at center one, traditional autonomic testing was performed along with electrogastrogram evaluation in a laboratory environment; at center two, 24-h Holter monitoring for heart rate variability by power-spectrum analysis was used. Other investigators have applied heart rate variability monitoring as a surrogate measure to reflect the visceral autonomic milieu in studies of hepatic and gastrointestinal (GI) illnesses [15-17]. At center two, we utilized heart rate variability, as measured by the frequency domain ratio of low-frequency/high-frequency power, to discriminate adrenergic/cholinergic balance as a measure of autonomic dysfunction [18].

Systemic autonomic testing data were analyzed for those patients enrolled in protocols for gastric neuromodulation. These protocols, inclusion and exclusion criteria, as well as patient characteristics are previously described in detail [19-21].

We also performed a pilot direct comparison of systemic autonomic testing and heart rate variability on a small number of patients undergoing electroacupuncture with an innovative electroacupuncture device. This pilot compared baseline heart rate variability with traditional autonomic testing in a small number of patients undergoing treatment with a transcutaneous electroacupuncture system.

\section{Study design}

The group at study center consisted of 39 patients (six males and 33 females, mean age 38 years) representing a subset of the gastric electro-mechanical stimulation study group [19] and the worldwide anti-vomiting electrical stimulation study trials [20]; patient diagnoses were diabetes mellitus $(n=11)$ and idiopathic disease $(\mathrm{n}=28)$. The second group of 35 patients (seven males and 28 females, mean age 37 years), with diagnoses of diabetes mellitus $(n=8)$ and idiopathic disease $(n$ $=27$ ) was selected from either the compassionate use electrical stimulation study [21] or from patients who were implanted under the humanitarian device exemption protocol established by the Food and Drug Administration in 2000.

The first group of 39 patients underwent traditional ANS evaluation, as well as enteric nervous system evaluation by cutaneous electrogastrography at baseline and at 1 year postgastric neuromodulation. Cutaneous electrogastrogram was recorded for at least $30 \mathrm{~min}$ in the resting position. The technique of electrogastrography has been previously described [13]. Cholinergic function was evaluated using two methods: changes in the R-to- $\mathrm{R}$ interval in response to three cycles of deep respiration; and the Valsalva ratio, represented by the change in heart rate in response to forced respiration for 15 $\mathrm{s}$ with a constant pressure of $40 \mathrm{~mm} \mathrm{Hg}$. Both the R-to- $\mathrm{R}$ interval and the Valsalva maneuver are summed and reported as cardio-vagal cholinergic function. Adrenergic arch function was assessed using an infrared photoplethysmography attached to the patient's index or middle finger. Photoplethysmography provides an indirect measure of arterial capillary blood flow. After baseline recordings, two parameters of capillary vasoconstriction reflexes in response to cold immersion and postural changes are elicited. The first adrenergic reflex, percentage of cutaneous vasoconstriction entails immersion of one hand for $1 \mathrm{~min}$ in ice-cold water, while the change in peripheral circulation in the opposite hand is measured. The second adrenergic parameter, postural adjustment ratio of capillary blood flow, is taken, with the patient seated, by measuring and recording changes in peripheral circulation in response to limb-posture changes relative to the level of the heart. Values from the adrenergic tests are added together and reported as sympathetic function. System autonomic testing protocols used at center one's autonomic laboratory have been previously described in detail [10-13]. In addition, a pool of age-related normative data has been collected and used in evaluating results. All autonomic measures were conducted on patients at baseline within 1 month prior to implanting the device and subsequently 1 year post-implantation at center laboratory where the autonomic and enteric nervous system measures were performed.

The second group of 35 patients was measured for autonomic balance by undergoing 24-h cardiac Holter monitoring of heart rate variability at baseline and post-implantation. 
Table 1. Autonomic and Enteric Measures at Baseline and 1 Year Post-Gastric Electrical Stimulation at Center One

\begin{tabular}{llll} 
Group 1: 39 patients & Baseline & Follow-up & P-value \\
\hline Gastroparesis (Gp) symptoms & $40.5 \pm 1.8$ & $25.4 \pm 2.9$ & $<0.001$ \\
Cardio-vagal function (VCF) & $21.9 \pm 2.3$ & $26.2 \pm 3.4$ & 0.15 \\
Sympathetic-adrenergic function (SAF) & $104.7 \pm 5.7$ & $82.9 \pm 8.4$ & $<0.05$ \\
Electrogastrography (EGG) & $3.8 \pm 0.2 \mathrm{cpm}$ & $3.6 \pm 0.1 \mathrm{cpm}$ & 0.3 \\
\hline
\end{tabular}

Heart rate variability, as measured by the time domain ratio of low-frequency/high-frequency power, reflects adrenergic/ cholinergic balance. Patients were seen at center two cardiology laboratories for fitting of the Holter monitor and follow-up Holter data collection/evaluation. The results were measured as the percentage of change of the maximum and minimum heart rate and were then compared with paired $t$-tests and reported as mean \pm standard error (SE).

Total symptom scores are expressed as the sum of scores as rated by the patient on a scale from 0 to 10 ( 0 being asymptomatic and 10 representing maximum severity) on the following symptoms: nausea, vomiting, anorexia/early satiety, bloating and/or distention and abdominal pain [21]. Data from standardized gastric emptying tests were not available on all patients due to differences in protocol requirements and thus, were not reviewed for this report. Results were compared by paired $t$-test and reported as mean $\pm \mathrm{SE}$.

The pilot group of electroacupuncture patients had both traditional systemic autonomic testing and heart rate variability. These five patients were studied at baseline before undergoing another stimulation therapy but did not have further testing of these two methodologies. This pilot study enabled us to compare systemic autonomic testing and heart rate variability in patients at baseline before undergoing non-invasive neurostimulation therapy.

\section{Results}

\section{Gastric neuromodulation and autonomic function testing associations}

For the first group of 39 patients at center one, systemic autonomic testing demonstrated an increase in cholinergic function (mean cardio-vagal cholinergic function $21.9 \pm 2.3$ at baseline vs. $26.2 \pm 3.4$ at 1 year, $\mathrm{P}=0.15)$ and decrease in adrenergic function (mean sympathetic adrenergic function $104.7 \pm 5.7$ at baseline vs. $82.9 \pm 8.4$ at 1 year, $\mathrm{P}<0.05)$, as well as some normalization in the electrogastrogram (mean $3.8 \pm 0.2 \mathrm{cpm}$ at baseline vs. $3.6 \pm 0.1 \mathrm{cpm}$ at 1 year, $\mathrm{P}=0.3$ (normal 3.0 - 3.3)). GI symptom scores decreased from 40.5 \pm 1.8 at baseline to $25.4 \pm 2.9$ at 1 year $(\mathrm{P}<0.001)$ (Table 1 and Fig. 1).

For the second group, at center two, of 35 patients, gastric neuromodulation use was associated with a decrease in lowfrequency/high-frequency ratio (baseline $1.4 \pm 0.1 \mathrm{vs.} \mathrm{follow-}$ up $1.1 \pm 0.07, \mathrm{P}=0.1$ ) indicating an increased cholinergic and/ or decreased adrenergic balance. GI symptoms decreased from $39.0 \pm 1.9$ at baseline to $19.9 \pm 3.7$ at follow-up $(\mathrm{P}<0.001)$ (Table 2 and Fig. 2).

The third group, a pilot study of five patients at center

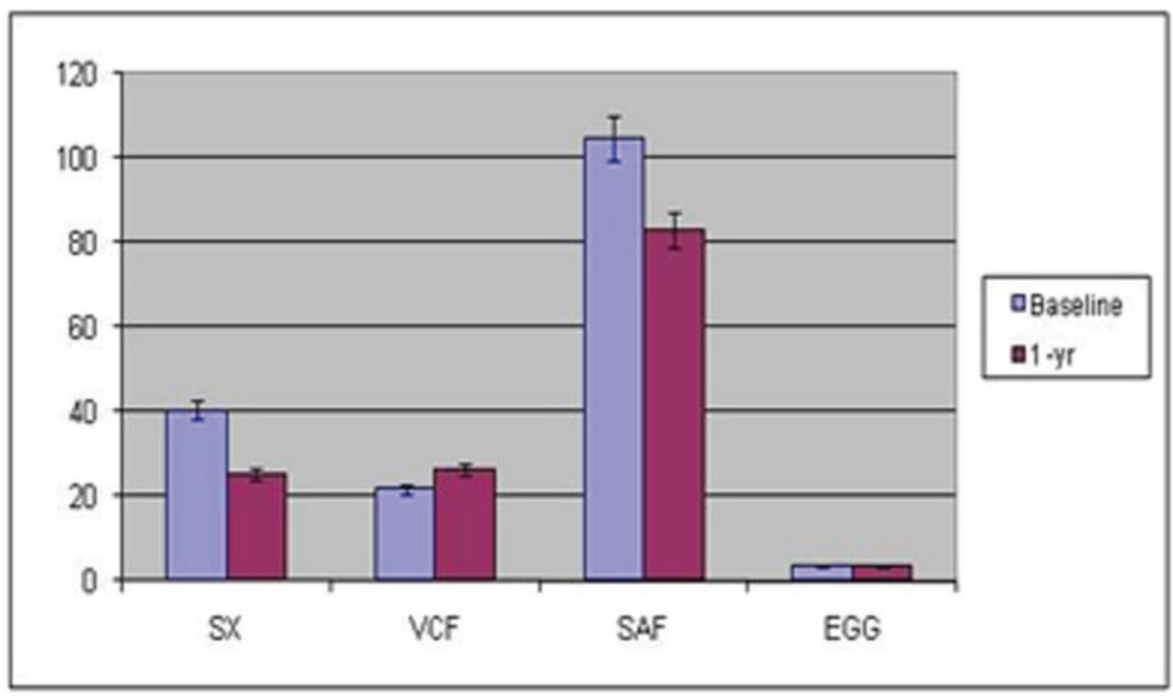

Figure 1. Symptoms, autonomic and enteric measures at baseline and 1 year after gastric stimulation at center one. EGG: electrogastrography; Gp: gastroparesis; SAF: sympathetic adrenergic function; VCF: vagal cholinergic function. 
Table 2. Symptoms and Heart Rate Variability in Patients Undergoing Gastric Electrical Stimulation at Center Two

\begin{tabular}{llll}
\hline Group 2: 35 patients & Baseline & Follow-up & P-value \\
\hline Symptoms & $39.0 \pm 1.9$ & $19.9 \pm 3.7$ & $<0.001$ \\
Low to high frequency ratio & $1.4 \pm 0.1$ & $1.1 \pm 0.07$ & 0.1 \\
\hline
\end{tabular}

three, revealed similar findings. Results of the comparison between systemic autonomic testing and heart rate variability in patients with the symptoms of gastroparesis are noted in Table 3 and Figure 3, including both quantitative and qualitative values. There was an $80 \%$ concordance between overall qualitative measurements but the quantification for sympathetic/parasympathetic measures was different. Also, cutaneous electrogastrogram recordings changed with other systemic autonomic testing, such as tilting.

\section{Discussion}

\section{Possible mechanisms from autonomic neuromodulation and autonomic function testing}

The mechanism of action of gastric neuromodulation has been the subject of some controversy. Previously we proposed several possible mechanisms of action for gastric neuromodulation [22], as have others. Such mechanisms include direct effect on gastric electrical rhythm disturbances or change in gastric emptying for liquids or solids [21]. Other possible mechanisms include the effect on fundic tone [23], or through modulation in the central nervous system [24]. While some evidence exists for most of the hypotheses, none have been shown conclusively to be the mechanism of action.

In this preliminary report, autonomic dysfunction found in severe gastroparesis patients was restored to a more normal basal autonomic balance in tandem with clinical improvement in classical symptoms of gastroparesis, primarily nausea and vomiting, in association with the intervention of gastric neuromodulation. Although we have not reported on controls, the difference in our current work lies in the mechanism(s) offered for the clinical improvement observed in our patients. Other evidence for an autonomic mediated response to gastric neuromodulation has been reported in a study of pancreatic exocrine function [25]. The stimulation parameters used in gastric neuromodulation, which are in the general energy range of neural stimulation, were originally based on limited animal data, and experience in one human $[26,27]$. Subsequent animal data have confirmed that those original parameters remain valid [28].

One proposed mechanism is that gastric neuromodulation may work via the ANS and was explored here by traditional autonomic measures along with the electrogastrogram. Using these data for analysis, evidence exists at follow-up for the effects of gastric neuromodulation on the ANS for both cholinergic augmentation and for adrenergic blockade. In addition, a slight normalization of the enteric nervous system measure of electrogastrogram appears to coincide with the above systemic autonomic testing changes.

Another exploration of the possible autonomic mechanism was performed by analyzing 24-h heart rate variability by power-spectrum analysis to reflect sympathovagal balance. Reported here, the most consistent findings of heart rate variability are those of an elevated ratio at baseline and a lowered ratio at follow-up, indicating a decrease in sympathetic adrenergic tone and/or an increase in vagal cholinergic tone.

Recent brain imaging reports [29] looking for a centrally mediated action of neuromodulation are not inconsistent with the changes in autonomic function proposed by our work, and indeed, would coincide with central changes also reported in the literature.

In general, our experience with systemic autonomic test-

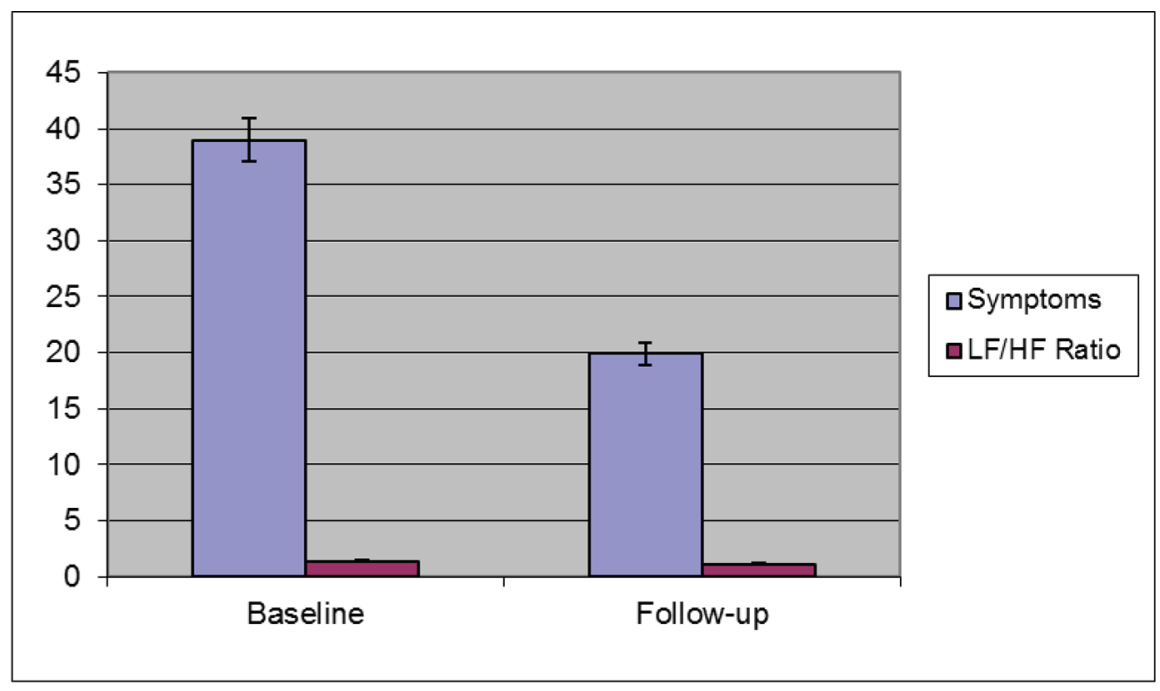

Figure 2. Symptoms and heart rate variability measures in patients undergoing gastric electrical stimulation at center two. LF: adrenergic/low-frequency; HF: cholinergic/high-frequency. 
Table 3. Comparison of Heart Rate Variability and Autonomic Nervous System With Electrogastrogram Values in a Pilot Study of Five Patients at Center Three

\begin{tabular}{|c|c|c|c|c|c|c|c|c|}
\hline Subject & $\begin{array}{l}\text { HRV \% } \% \text { LF/ } \\
\text { HF with meal }\end{array}$ & $\begin{array}{l}\text { HRV } \\
\text { interpretation }\end{array}$ & $\begin{array}{l}\text { SAF } \\
\% \mathrm{VC}\end{array}$ & $\begin{array}{l}\text { SAF } \\
\text { PAR }\end{array}$ & $\begin{array}{l}\text { Total SAF = } \\
\text { VC + PAR }\end{array}$ & $\begin{array}{l}\text { Total VCF }=\text { RR } \\
+\mathrm{VR}+\mathbf{3 0} / \mathbf{1 5}\end{array}$ & $\begin{array}{l}\text { AFT } \\
\text { interpretation }\end{array}$ & $\begin{array}{l}\text { EGG } \% \Delta \text { in } \\
\text { frequency with meal }\end{array}$ \\
\hline 1 & 33.33 & $\begin{array}{l}\text { Sympathetic } \\
\text { dominant }\end{array}$ & 92 & 3.3 & 95.30 & 20.23 & $\begin{array}{l}\text { Normal } \\
\text { parasympathetic/ } \\
\text { abnormal } \\
\text { sympathetic (PAR) }\end{array}$ & -24.10 \\
\hline 2 & 21.57 & $\begin{array}{l}\text { Sympathetic } \\
\text { dominant }\end{array}$ & 79 & 2.2 & 81.20 & 14.28 & $\begin{array}{l}\text { Abnormal } \\
\text { sympathetic (PAR) }\end{array}$ & 79.86 \\
\hline 4 & -42.62 & $\begin{array}{l}\text { Parasympathetic } \\
\text { dominant }\end{array}$ & 84.5 & 3.5 & 88.00 & 9.13 & $\begin{array}{l}\text { Abnormal } \\
\text { parasympathetic } \\
>\text { abnormal } \\
\text { sympathetic (PAR) }\end{array}$ & -18.37 \\
\hline 5 & 92.00 & $\begin{array}{l}\text { Sympathetic } \\
\text { dominant }\end{array}$ & 98 & 14 & 112.00 & 20.61 & $\begin{array}{l}\text { Normal } \\
\text { parasympathetic/ } \\
\text { abnormal } \\
\text { sympathetic (PAR) }\end{array}$ & -27.88 \\
\hline
\end{tabular}

AFT: autonomic nervous system testing; EGG: electrogastrography; Gp: gastroparesis; HF: cholinergic/high frequency; HRV: heart rate variability; LF: adrenergic/low-frequency; SAF: sympathetic adrenergic function; VCF: vagal cholinergic function.

ing in gastroparesis patients has reinforced the importance of using multiple measures of autonomic function. Single indices are not meaningful, especially considering the complex array of neuronal autonomic pathways needed for gastric motility functioning. In particular, adrenergic tests of vasoconstriction to cold stress and postural-adjustment ratio have been found to correlate to other clinical measures in studying gastric motor dysfunction and other chronic vomiting syndromes.

Comparison of heart rate variability with traditional autonomic testing, as in our pilot study at baseline, showed similarities between both methods of autonomic testing in response to electroacupuncture.

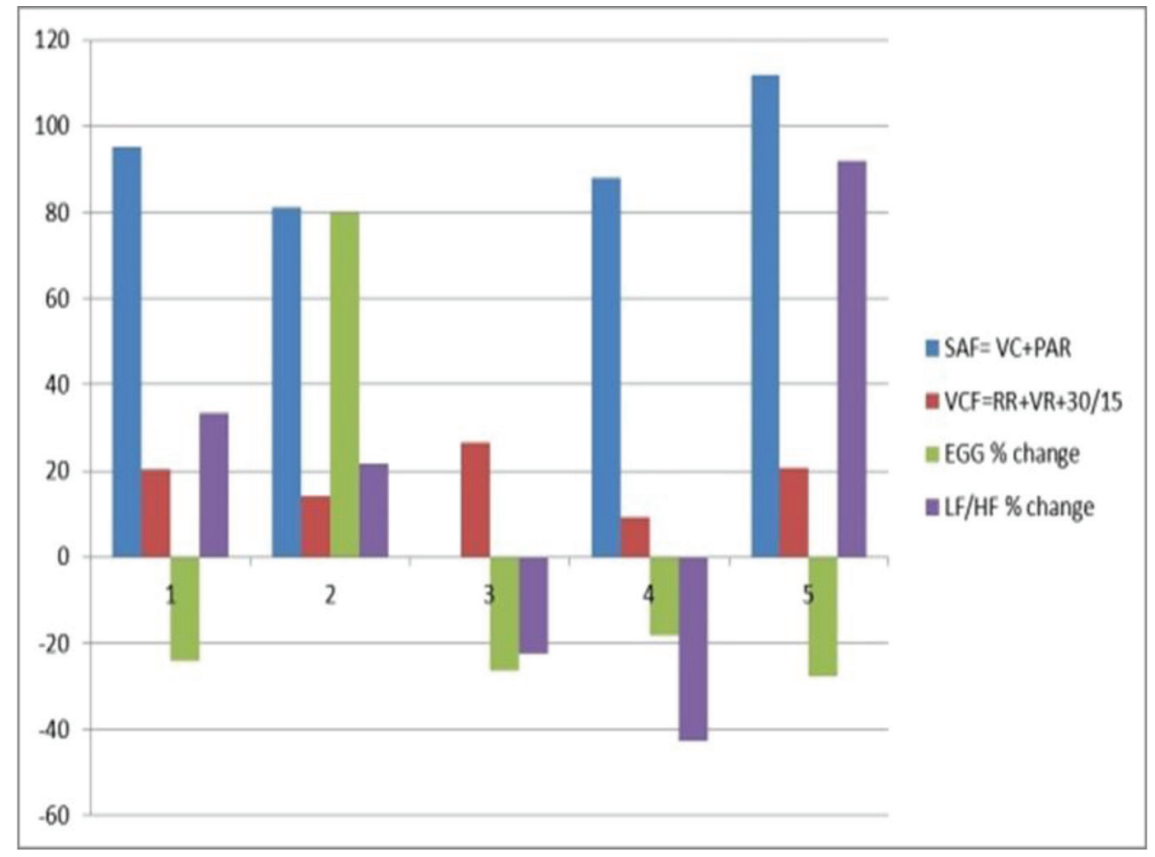

Figure 3. Comparison of autonomic function testing and heart rate variability in five patients with diabetic gastroparesis at baseline at center three. VCF: vagal cholinergic function; RR: R-to-R interval; VR: Valsalva ratio; SAF: sympathetic adrenergic function; VC: vasoconstriction; PAR: postural adjustment ratio; EGG: electrogastrography; LF: adrenergic/low-frequency; HF: cholinergic/high-frequency; HRV: heart rate variability. 
Both methods have been used previously to evaluate gastroparesis but have never been compared directly. In our pilot study, we performed both methods of autonomic testing. Five patients with diabetic gastroparesis underwent both systemic autonomic testing and heart rate variability (with electrogastrogram) testing. We demonstrated a good correlation of systemic autonomic testing and heart rate variability testing with $80 \%$ concordance between overall qualitative measurements.

\section{Future for GI neurostimulation and autonomic function studies}

Future work on the mechanism of gastric neuromodulation needs to include prospective studies of simultaneous measurements of the enteric, autonomic and central nervous systems, including measurements of gastric fundic pressure, liquid and solid gastric emptying, and pyloric pressure. A search for correlations of these measurements to symptom scores and quality-of-life questionnaires could also help extend this work into possible predictors of outcome.

The advent of temporary endoscopic placement of gastric neuromodulation devices may allow for these simultaneous measures in controls as well as providing a variety of patients, thus offering further insights into possible mechanism(s) of action [30].

In addition, the availability of non-invasive therapies for neurostimulation, such as the pilot of electroacupuncture used here, may offer a number of new therapeutic options. Both traditional systemic autonomic testing and heart rate variability testing may offer opportunities for understanding the mechanisms of action of these therapies.

As with testing for other disorders, heart rate variability may be useful as a screen, with more detailed testing being used as a confirmatory test.

\section{Non-technical summary}

Disorders of the upper GI track often present with nausea vomiting and abdominal pain. These symptoms are proposed to originate from neuromuscular etiologies, with autonomic manifestation, and often respond to electrical stimulation, both implanted and also non-implanted devices, such as electroacupuncture. We have summarized results of ANS tests - both traditional autonomic testing and cardiac heart rate variability - at baseline and after gastric neuromodulation as well as results from baseline with electroacupuncture.

\section{Conclusion}

Our pilot study showed that both systemic autonomic testing and heart rate variability testing can be performed for patients with the symptoms of gastroparesis undergoing two types of neurostimulation therapies. The clinical usefulness of ANS measures and their response to neurostimulation therapies will need to be determined in future clinical studies.

\section{Acknowledgement}

The authors would like to thank the staffs of autonomic function testing lab at UT-Memphis, the Cardiology Holter Laboratory and GI Lab at UAMS; Warren Starkebaum for helpful suggestions in the manuscript, and Nighat Abidi for help with statistical calculations and for manuscript preparation. We would also like to thank Jeanne Keith-Ferris and Elizabeth Gooden for their review of this manuscript and recommendations. Finally, we would like to thank Cecelia Delbridge Troy Berch, Kristin Mallard and the staff of the GI Division at UMMC, Jackson, MS. We would also like to thank the staff of the GI Division at the University of Louisville, especially Ed Miller and Catherine McBride, as well as the help of Dr. Jiande Chen's laboratory at UTMB in Galveston.

\section{Disclosures and Funding}

Dr. Abell is a consultant, licensor and investigator with Medtronic; certain aspects of the technology used are covered by intellectual property assigned from the University of Mississippi to ADEPT-GI.

\section{References}

1. Abell TL, Van Cutsem E, Abrahamsson H, Huizinga JD, Konturek JW, Galmiche JP, VoelIer G, et al. Gastric electrical stimulation in intractable symptomatic gastroparesis. Digestion. 2002;66(4):204-212.

2. Lin Z, Sarosiek I, Forster J, McCallum RW. Symptom responses, long-term outcomes and adverse events beyond 3 years of high-frequency gastric electrical stimulation for gastroparesis. Neurogastroenterol Motil. 2006;18(1):1827.

3. McCallum RW, Dusing RW, Sarosiek I, Cocjin J, Forster $\mathrm{J}$, Lin Z. Mechanisms of symptomatic improvement after gastric electrical stimulation in gastroparetic patients. Neurogastroenterol Motil. 2010;22(2):161-167, e150161.

4. Anand C, Al-Juburi A, Familoni B, Rashed H, Cutts T, Abidi N, Johnson WD, et al. Gastric electrical stimulation is safe and effective: a long-term study in patients with drug-refractory gastroparesis in three regional centers. Digestion. 2007;75(2-3):83-89.

5. Mason RJ, Lipham J, Eckerling G, Schwartz A, Demeester TR. Gastric electrical stimulation: an alternative surgical therapy for patients with gastroparesis. Arch Surg. 2005;140(9):841-846; discussion 847-848.

6. Forster J, Damjanov I, Lin Z, Sarosiek I, Wetzel P, McCallum RW. Absence of the interstitial cells of Cajal in patients with gastroparesis and correlation with clinical findings. J Gastrointest Surg. 2005;9(1):102-108.

7. Zarate N, Mearin F, Wang XY, Hewlett B, Huizinga JD, Malagelada JR. Severe idiopathic gastroparesis due to neuronal and interstitial cells of Cajal degeneration: pathological findings and management. Gut. 2003;52(7):966- 
970.

8. Islam S, Vick LR, Runnels MJ, Gosche JR, Abell T. Gastric electrical stimulation for children with intractable nausea and gastroparesis. J Pediatr Surg. 2008;43(3):437442 .

9. Muth ER, Koch KL, Stern RM. Significance of autonomic nervous system activity in functional dyspepsia. Dig Dis Sci. 2000;45(5):854-863.

10. Abell TL, Cardoso S, Schwartzbaum J, Familoni B, Wilson R, Massie D. Diabetic gastroparesis with an abnormality in sympathetic innervation. Eur J Gastroenterol Hepatol. 1994;6:241-247.

11. Rashed H, Abell TL, Familoni BO, Cardoso S. Autonomic function in cyclic vomiting syndrome and classic migraine. Dig Dis Sci. 1999;44(8 Suppl):74S-78S.

12. Rashed H, Cutts T, Abell T, Cowings P, Toscano W, ElGammal A, Adl D. Predictors of response to a behavioral treatment in patients with chronic gastric motility disorders. Dig Dis Sci. 2002;47(5):1020-1026.

13. Hathaway DK, Abell T, Cardoso S, Hartwig MS, el Gebely S, Gaber AO. Improvement in autonomic and gastric function following pancreas-kidney versus kidney-alone transplantation and the correlation with quality of life. Transplantation. 1994;57(6):816-822.

14. Rahman F, Pechnik S, Gross D, Sewell L, Goldstein DS. Low frequency power of heart rate variability reflects baroreflex function, not cardiac sympathetic innervation. Clin Auton Res. 2011;21(3):133-141.

15. Verne GN, Soldevia-Pico C, Robinson ME, Spicer KM, Reuben A. Autonomic dysfunction and gastroparesis in cirrhosis. J Clin Gastroenterol. 2004;38(1):72-76.

16. Keresztes K, Istenes I, Folhoffer A, Lakatos PL, Horvath A, Csak T, Varga P, et al. Autonomic and sensory nerve dysfunction in primary biliary cirrhosis. World J Gastroenterol. 2004;10(20):3039-3043.

17. Heitkemper M, Jarrett M, Cain KC, Burr R, Levy RL, Feld A, Hertig V. Autonomic nervous system function in women with irritable bowel syndrome. Dig Dis Sci. 2001;46(6):1276-1284.

18. Heart rate variability. Standards of measurement, physiological interpretation, and clinical use. Task Force of the European Society of Cardiology and the North American Society of Pacing and Electrophysiology. Eur Heart J. 1996;17(3):354-381.

19. The GEMS study group. Report of a multicenter study on electrical stimulation for the treatment of gastroparesis. Gastroenterology. 1997;112(4):A735.

20. Abell T, McCallum R, Hocking M, Koch K, Abrahamsson $\mathrm{H}$, Leblanc I, Lindberg G, et al. Gastric electrical stimulation for medically refractory gastroparesis. Gastroenterology. 2003;125(2):421-428.

21. Lin Z, Forster J, Sarosiek I, McCallum RW. Effect of high-frequency gastric electrical stimulation on gastric myoelectric activity in gastroparetic patients. Neurogastroenterol Motil. 2004;16(2):205-212.

22. Luo J, TL Abell, P Eaton, J Familoni, H Rashed, G Voeller: Gastric Electrical Stimulation: We Know Its Effect, But What Is The Mechanism? Gastroenterology 1999;116(4) (Part 2):A1033.

23. Tack J, Coulie B, Van Cutsem E, Ryden J, Janssens KU. The influence of gastric electrical stimulation on proximal gastric motor and sensory function in severe idiopathic gastroparesis. Gastroenterology 1999;116(4):G4733.

24. Tang M, Zhang J, Chen JD. Central mechanisms of gastric electrical stimulation involving neurons in the paraventricular nucleus of the hypothalamus in rats. Obes Surg. 2006;16(3):344-352.

25. Luo J, Al-Juburi A, Rashed H, O'Dorisio T, Marchal B, Starkebaum W, Abell TL. Gastric electrical stimulation is associated with improvement in pancreatic exocrine function in humans. Pancreas. 2004;29(2):e41-44.

26. Familoni BO, Abell TL, Voeller G, Salem A, Gaber O. Electrical stimulation at a frequency higher than basal rate in human stomach. Dig Dis Sci. 1997;42(5):885-891.

27. Familoni BO, Abell TL, Nemoto D, Voeller G, Johnson B. Efficacy of electrical stimulation at frequencies higher than basal rate in canine stomach. Dig Dis Sci. 1997;42(5):892-897.

28. Le Blanc-Louvry I, Guerre F, Songne B, Ducrotte P. Gastric stimulation: influence of electrical parameters on gastric emptying in control and diabetic rats. BMC Surg. 2002;2:5.

29. Qin C, Sun Y, Chen JD, Foreman RD. Gastric electrical stimulation modulates neuronal activity in nucleus tractus solitarii in rats. Auton Neurosci. 2005;119(1):1-8.

30. Ayinala S, Batista O, Goyal A, Al-Juburi A, Abidi N, Familoni B, Abell T. Temporary gastric electrical stimulation with orally or PEG-placed electrodes in patients with drug refractory gastroparesis. Gastrointest Endosc. 2005;61(3):455-461. 